\title{
Experiment and computation: a combined approach to study the van der Waals complexes
}

\author{
L.A. Surin* \\ Institute for Spectroscopy RAS, 108840 Troitsk, Moscow, Russia
}

\begin{abstract}
A review of recent results on the millimetre-wave spectroscopy of weakly bound van der Waals complexes, mostly those which contain $\mathrm{H}_{2}$ and $\mathrm{He}$, is presented. In our work, we compared the experimental spectra to the theoretical bound state results, thus providing a critical test of the quality of the $\mathrm{M}-\mathrm{H}_{2}$ and $\mathrm{M}-\mathrm{He}$ potential energy surfaces (PESs) which are a key issue for reliable computations of the collisional excitation and de-excitation of molecules $\left(\mathrm{M}=\mathrm{CO}, \mathrm{NH}_{3}, \mathrm{H}_{2} \mathrm{O}\right)$ in the dense interstellar medium. The intermolecular interactions with $\mathrm{He}$ and $\mathrm{H}_{2}$ play also an important role for high resolution spectroscopy of helium or para-hydrogen clusters doped by a probe molecule $(\mathrm{CO}, \mathrm{HCN})$. Such experiments are directed on the detection of superfluid response of molecular rotation in the $\mathrm{He}$ and $p-\mathrm{H}_{2}$ clusters.
\end{abstract}

The presence of molecular species like $\mathrm{CO}, \mathrm{H}_{2} \mathrm{O}, \mathrm{NH}_{3}$ in the interstellar medium (ISM) and their usefulness as indicators of molecular cloud properties has motivated considerable experimental and theoretical efforts aimed largely at understanding the interactions between these species and their main ISM collision partners, $\mathrm{H}_{2}$ and $\mathrm{He}[1,2]$. The collisional excitations of the species involved strongly depend on important features of the multidimensional intermolecular potential energy surfaces (PES), such as barriers, anisotropies, binding energy, and structure in the most stable configurations.

High resolution spectroscopy of van der Waals complexes has proofed to be a very effective tool for the elucidation of intermolecular PES, because the bound states of the complexes are sensitive to the interaction potential [3]. The weakly bound complexes can be efficiently produced in a supersonic jet expansion of a gas mixture into vacuum. A difficulty in undertaking the spectral measurements of such systems is that the transitions are extremely weak. The recent progress on the millimeter-wave studies of weakly bound molecular complexes, mostly those which contain $\mathrm{H}_{2}$ and $\mathrm{He}$, using intracavity OROTRON jet spectrometer [4] along with double resonance technique is presented. These experimental measurements provided rigorous tests of the accuracy of the ab initio PESs and computed bound states $[5,6]$.

Another application, in which the intermolecular interactions with $\mathrm{He}$ and $\mathrm{H}_{2}$ play an important role, is the doping of helium or para-hydrogen clusters with a probe molecule to perform high resolution spectroscopy. Such experiments are directed on the detection of superfluid response of molecular rotation in the $\mathrm{He}$ and $p-\mathrm{H}_{2}$ clusters [7]. It was shown

*Corresponding author: surin@isan.troitsk.ru 
recently that the lighter probe molecules $(\mathrm{CO}, \mathrm{HCN})$ allow one to measure the effective inertia of $\mathrm{He}$ and $p-\mathrm{H}_{2}$ clusters while maintaining a maximum superfluid response with respect to dopant rotation.

\section{References}

1. S. Green, P. Thaddeus, Astrophys. J. 205, 766 (1976)

2. S. Chefdeville, T. Stoecklin, C. Naulin, P. Jankowski, K. Szalewicz, A. Faure, M. Costes, A. Bergeat, Astrophys. J. Lett. 799, L9 (2015)

3. P.E.S. Wormer, A. van der Avoird, Chem. Rev. 100, 4109 (2000)

4. L.A. Surin, B.S. Dumesh, F. Lewen, D.A. Roth, V.P. Kostromin, F.S. Rusin, G. Winnewisser, I. Pak, Rev. Sci. Instrum. 72, 2535 (2001)

5. P. Jankowski, L. Surin, A. Potapov, S. Schlemmer, A.R.W. McKellar, K. Szalewicz, J. Chem. Phys. 138, 084307 (2013)

6. A.V. Potapov, L.A. Surin, V.A. Panfilov, B.S. Dumesh, T.F. Giesen, S. Schlemmer, P.L. Raston, W. Jäger, Astrophys. J. 703, 2108 (2009)

7. L.A. Surin, JETP Lett. 97, 57 (2013) 